\title{
Certain Problems of the Circuit Test Diagnosis
}

\author{
Nikolay V. Kinsht", Natalia N. Petrun'ko \\ Institute of Automation and Control Processes Far East Branch Russian Academy of Science, Vladivostok, 690041, Russia \\ *Corresponding Author: kin@dvo.ru
}

Copyright $@ 2013$ Horizon Research Publishing All rights reserved.

\begin{abstract}
Certain problems of test diagnosing of electric circuit on the basis of application of a matrix which elements are linearly related with the desired internal parameters are considered. Use of redundant experiments is presented. Analytical solutions of a task when the part of required parameters is known and way of diagnosis of multipole network with inaccessible nodes are received. Examples are showed.
\end{abstract}

Keywords Circuit, Diagnostic Model, Multipole, Testing, Accessible Nodes, Inaccessible Nodes

\section{Introduction}

Based on achievements of the classical circuit theory, one of the most natural initial approaches to the diagnostics of electric chains is representation of diagnosed circuit in the form of a passive multipole network. Parameters of this multipole network are subject to definition. The pioneer in statement and systematic studying of such task is Berkowitz and his colleagues ([1], et al.). In its statement possibility of diagnostics of the passive multipole network is considered. Some of nodes are considered available both for giving of entrance signals, and for measurement of reactions, some of nodes are partially available on measurements of reactions only, and other nodes are inaccessible for any connections. Its works found a number of followers and are realized in a significant amount of patents of the USA ([2] et al.).

The criterion for the solution of the diagnosis is to determine the numerical values of the internal parameters of the elements diagnosed circuit (resistors, capacitors, etc.). Actuality of the problem in general has caused a lot of publications in which the authors refined setting goals and devised various methods of solutions. There are a number of detailed reviews of the problem, it isn't a goal this paper. For example, the main areas of research it is correctly described in [3].

One of the most difficulties of the solution problem lies in the fact that the analytic relationship between the measurements and the required elements parameters are non-linear even for linear resistive circuits. Therefore, the use of symbolic model known methods [4] or, for example, fault dictionaries [5] are known. In [3], for example, changes in the internal resistance parameters modeled by additional current sources.

Considerable interest to diagnostics of a passive multipole at various assumptions was shown by the Russian researches [6]. Problems of circuit diagnostics are for a long time a subject of researches of one of authors of the present article $[7,8]$. One of the successful principles of the mathematical diagnosis model is using as the basis a circuit matrix, which elements are linearly related with the desired internal parameters. Here may be used various ways solutions nodal analysis, loop analysis and other. The study of this set of problems is of scientific interest to present.

\section{The Principal Model Diagnosis of Electric Circuits}

\subsection{Basic Equations}

Let's consider a passive multipole network $\Pi$ with $(n+1)$ nodes. First $\mathrm{n}$ nodes are independent. Let us turn to the problem of determining the inner parameters and as diagnostic model we will take circuit nodal admittance matrix $\mathbf{Y}_{n}$.

In accordance with the nodal circuit analysis the fundamental equations for the electric conditions analysis (in the matrix form) look as:

$$
\mathbf{Y}^{n} \mathbf{U}=\mathbf{J},
$$

where $\mathbf{U}=\operatorname{col}\left[U_{1}, \ldots U_{\mathrm{n}}\right], \mathbf{J}=\operatorname{col}\left[J_{1}, \ldots J_{\mathrm{n}}\right]$ are the vectors of nodal voltages and nodal currents, respectively, and $\mathbf{Y}^{n}$ is the square matrix of nodal immitances.

It is clearly, that if there are no parallel branches in the circuit, then the structure and the numerical value of the immitances of all the branches may be easy restore based on the matrix $\mathbf{Y}^{n}$.

Let us organize testing experiments as an influence of current sources conducted on the $n$ nodes of the passive circuit П (Fig. 1).

There are $n$ nodal voltages vectors (relative to the base node) in each experiment:

$$
\mathbf{U}_{k}=\left(\mathbf{Y}^{n}\right)^{-1} \mathbf{J}_{k},(k=1 \ldots n) .
$$

Let express vectors of input currents of all the experiments as 
a square matrix of currents $\mathbf{J}_{\mathrm{R}}=\left[\mathbf{J}_{1}, \ldots, \mathbf{J}_{n}\right]$ and compose square matrix of voltages $\mathbf{U}_{\mathrm{R}}=\left[\mathbf{U}_{1}, \ldots, \mathbf{U}_{n}\right]$.

The desired nodal admittance matrix might then be written as:

$$
\mathbf{Y}^{n}=\mathbf{J}_{\mathrm{R}} \mathbf{U}_{\mathrm{R}}{ }^{-1}
$$

It is obvious that if part of the passive parameters (admittances) are known and expressed as nodal matrix $\mathbf{Y}_{0}$, the result of diagnostic test is the difference:

$$
\mathbf{Y}^{n}=\mathbf{J}_{\mathrm{R}} \mathbf{U}_{\mathrm{R}}{ }^{-1}-\mathbf{Y}_{0} .
$$

Similarly to foregoing approach we can formulate the problem in the other basis, for example, the dual formulation of loop analysis.

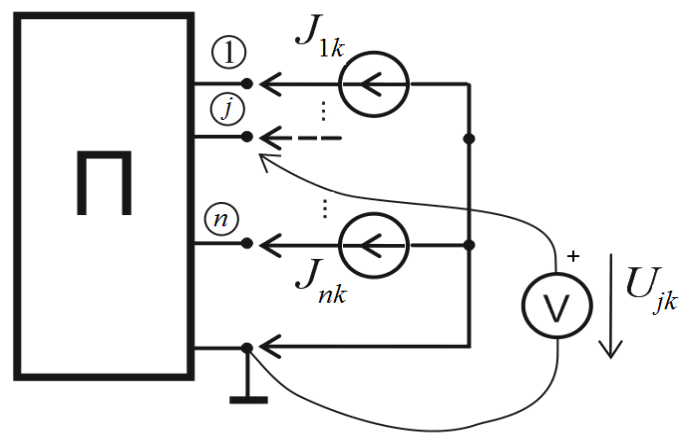

Figure 1. Testing experiments at passive circuit $\Pi$

\subsection{Example 1}

Let's illustrate the approach for alternating current circuit. Imagine that the multipole network with $n=4$ independent nodes has been tested by complex matrix of input actions (all values below are in the dimentionless form):

$$
\mathbf{J}_{R}=\left[\begin{array}{cccc}
1 & 2 & 0 & 0 \\
0.5 \mathrm{j} & 0 & \mathrm{j} & 0.5 \mathrm{j} \\
0 & 1 & 1+\mathrm{j} & 0 \\
0 & 0 & 0 & 1
\end{array}\right]
$$

Let imagine that as a result of measurements we obtain the complex-matrix of nodal voltages:

$$
\mathbf{U}_{R}=\left[\begin{array}{llll}
2.08 \angle 34^{\circ} & 4.13 \angle 16^{\circ} & 2.63 \angle 53^{\circ} & 0.41 \angle 29^{\circ} \\
1.06 \angle 10^{\circ} & 3.31 \angle-8^{\circ} & 3.07 \angle 84^{\circ} & 0.48 \angle 60^{\circ} \\
0.88 \angle 33^{\circ} & 3.51 \angle-15^{\circ} & 2.25 \angle 45^{\circ} & 0.21 \angle 115^{\circ} \\
0.32 \angle-39^{\circ} & 1.12 \angle-70^{\circ} & 0.85 \angle 12^{\circ} & 0.99 \angle-52^{\circ}
\end{array}\right]
$$

We calculate the values of the matrix of nodal admittances follow (2):

$$
\tilde{\mathbf{Y}}=\left[\begin{array}{cccc}
0.2890-0.5078 j & 0.0036+0.5072 j & 0.0037+0.0090 j & 0.0034+0.0069 j \\
0.0046+0.5106 j & 0.3623-0.1035 j & -0.0073-0.5117 j & -0.1961-0.0111 j \\
0.0111+0.0016 j & -0.0096-0.5132 j & 0.2441+0.5057 j & -0.1579-0.0006 j \\
0.0018-0.0067 j & -0.2048-0.0000 j & -0.1494+0.0092 j & 0.5514+0.8968 j
\end{array}\right] \approx
$$

$$
\left[\begin{array}{cccc}
(0.29-0.51 \mathrm{j}) & 0.51 \mathrm{j} & 0 & 0 \\
0.51 \mathrm{i} & (0.36-0.10 \mathrm{j}) & -0.51 \mathrm{j} & -0.19 \\
0 & -0.51 \mathrm{j} & 0.24+0.51 \mathrm{j} & -0.15 \\
0 & -0.20 & -0.15 & 0.55+0.9 \mathrm{i}
\end{array}\right] .
$$

And after rounding values of matrix elements it is easy to decipher the matrix, the off-diagonal terms are corresponding to the common node admittance and diagonal terms are the sum nodal admittance:

$$
\begin{gathered}
y_{12}=y_{21}=-0.51 \mathrm{j}, \quad y_{23}=y_{32}=0.51 \mathrm{j}, \\
y_{24}=y_{42}=-0.19, \quad y_{34}=y_{43}=0.15, \\
y_{15}=y_{51}=0.29, \quad y_{25}=y_{52}=0.17-0.09 \mathrm{j}, \\
y_{35}=y_{53}=0, \quad y_{45}=y_{54}=0.12+0.9 \mathrm{j} ;
\end{gathered}
$$

and testing circuit can be represent as showed on Fig. 2. Numerical values of circuit parameters are specified in drawing.

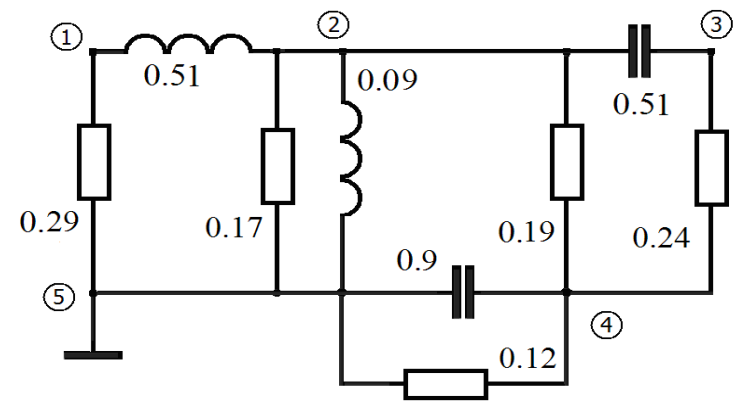

Figure 2. Circuit for Example 1

\subsection{Redundant Experiments}

Let's imagine that in order to reduce possible errors of measurements and calculations redundant experiments are made, and thus the matrix of input actions becomes a rectangular matrix. Here we use the idea of the pseudoinverse matrix. In accordance with the method, we multiply both sides of the right by transposed matrix of the measured voltages and resolve it relative to the required immitance matrix:

$$
\mathbf{Y}^{n}=\mathbf{J}_{\mathrm{R}} \quad \mathbf{U}_{\mathrm{R}}^{\mathrm{T}}\left(\mathbf{U}_{\mathrm{R}} \mathbf{U}_{\mathrm{R}}^{\mathrm{T}}\right)^{-1}
$$

\subsection{Example 2}

The conductivity of the branches in dimensionless units of an initial circuit are indicated in the Fig. 3. The circuit has 4 independent nodes, and its nodal conductance matrix may be expressed as:

$$
\mathbf{Y}^{n}=\left[\begin{array}{cccc}
4 & -2 & -1 & 0 \\
-2 & 10 & -2 & -5 \\
-1 & -2 & 7 & -4 \\
0 & -5 & -4 & 11
\end{array}\right]
$$




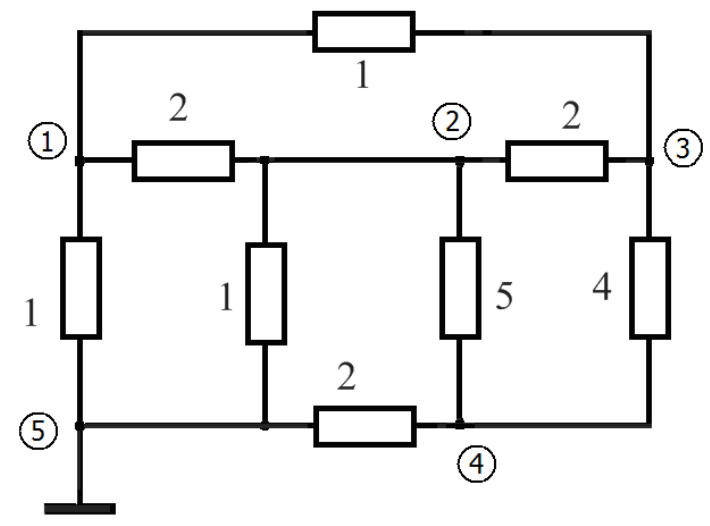

Figure 3. Circuit for Example 2

Let us draw up the matrix of input actions JR

$$
\mathbf{J}_{R}=\left[\begin{array}{cccccc}
10 & 0 & 0 & 0 & 5 & 1 \\
0 & 1 & 0 & 2 & 5 & 2 \\
0 & 5 & 1 & 0 & 5 & 3 \\
0 & 0 & 0 & 13 & 5 & 4
\end{array}\right]
$$

and then, for example, we may get the matrix of "measured" voltage nodes (up to three characters), columns of the matrix correspond to the individual experiments

$$
\mathbf{U}_{R}=\left[\begin{array}{llllll}
4.16 & 1.35 & 0.23 & 2.81 & 5.22 & 2.26 \\
2.20 & 1.58 & 0.25 & 3.67 & 5.10 & 2.54 \\
2.26 & 2.24 & 0.40 & 3.88 & 5.68 & 2.96 \\
1.82 & 1.53 & 0.26 & 4.26 & 4.84 & 2.60
\end{array}\right]
$$

Now in accordance with (4) we have:

$$
\tilde{\mathbf{Y}}^{n}=\left[\begin{array}{cccc}
4.0059 & -1.9804 & -1.0043 & -0.0215 \\
-2.0246 & 9.9413 & -1.9049 & -5.0230 \\
-1.0115 & -1.8266 & 6.8816 & -4.0258 \\
0.0333 & -5.1665 & -3.9536 & 11.0786
\end{array}\right],
$$

which is consistent with the original data.

\section{Active Multipole Circuit}

Above the task was to diagnose the parameters of passive multipole circuit. Imagine that there are independent sources inside, and the multipole circuit network $\mathrm{A}$ is an active with non-known sources (Fig. 4) Then to calculate the mode of circuit in the absence of testing actions we have:

$$
\mathbf{Y}^{n} \mathbf{U}_{0}=\mathbf{J}_{0},
$$

where are:

$\mathbf{J}_{0}$ - the vector of internal (non-known) sources, $\mathbf{U}_{0}=\operatorname{col}\left[U_{01}, \ldots U_{0 \mathrm{n}}\right]-$ the nodes voltages in the open-circuit conditions, as shown at Fig. 4.

For each $k$-th test experiment with source $\mathbf{J}_{k}(k=1, \ldots n)$ we can write:

$$
\begin{gathered}
\mathbf{Y}^{n} \mathbf{U}_{\mathrm{k}}=\left(\mathbf{J}_{0}+\mathbf{J}_{\mathrm{k}}\right)=\mathbf{Y}^{n} \mathbf{U}_{0}+\mathbf{J}_{\mathrm{k}}, \\
\mathbf{Y}^{n}\left(\mathbf{U}_{\mathrm{k}}-\mathbf{U}_{0}\right)=\mathbf{J}_{\mathrm{k}}
\end{gathered}
$$

Let's enter into consideration square matrix $\mathbf{U}_{0 \mathrm{R}}=\left[\mathbf{U}_{01}, \ldots \mathbf{U}_{0 \mathrm{n}}\right]$, and similarly (2) we have:

$$
\mathbf{Y}^{n}=\mathbf{J}_{\mathrm{R}}\left(\mathbf{U}_{\mathrm{R}}-\mathbf{U}_{0 \mathrm{R}}\right)^{-1} \text {. }
$$

We can also use other formal description, If we make $(n+1)$ different test inputs $\mathbf{J}_{1}, \ldots, \mathbf{J}_{n+1}$, we single out the vector of $n$ of independent increments

$$
\Delta \mathbf{J}_{\mathrm{R}}=\left[\left(\mathbf{J}_{1}-\mathbf{J}_{n+1}\right), \ldots,\left(\mathbf{J}_{n^{-}} \mathbf{J}_{n+1}\right)\right]=\left[\Delta \mathbf{J}_{1}, \ldots, \Delta \mathbf{J}_{n}\right],
$$

and determine the vector of variation of the nodal voltages

$$
\boldsymbol{\Delta} \mathbf{U}_{\mathrm{R}}=\left[\left(\mathbf{U}_{1}-\mathbf{U}_{n+1}\right), \ldots,\left(\mathbf{U}_{n}-\mathbf{U}_{n+1}\right)\right]=\left[\boldsymbol{\Delta} \mathbf{U}_{1}, \ldots, \boldsymbol{\Delta} \mathbf{U}_{n}\right],
$$

the expression $\mathbf{Y}^{n}$ can be written as

$$
\mathbf{Y}^{n}=\Delta \mathbf{J}_{\mathrm{R}} \Delta \mathbf{U}_{\mathrm{R}}{ }^{-1} \text {. }
$$

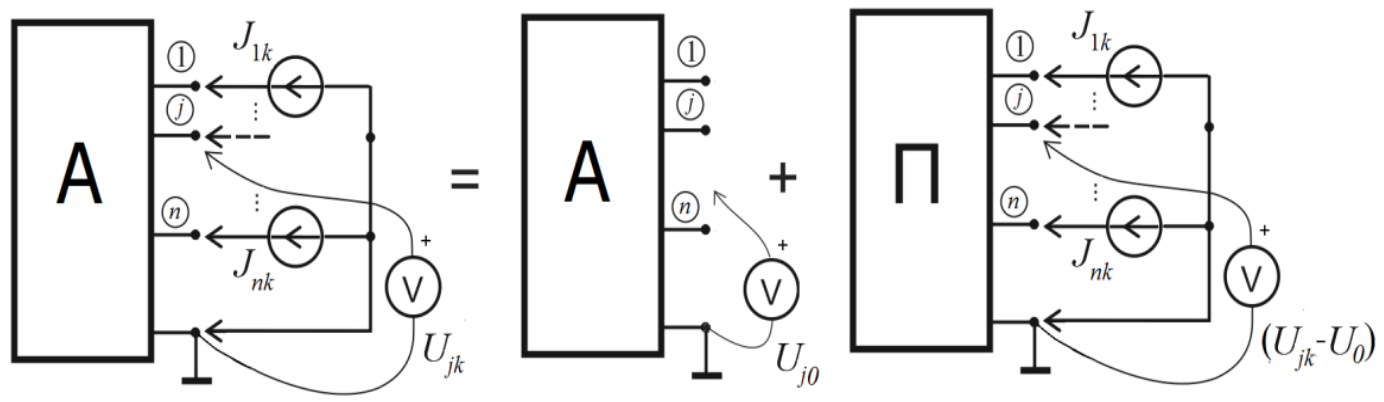

Figure 4. The active multipole circuit 


\section{Diagnosis of Multipole Network with Inaccessible Nodes}

\subsection{Basic Equations}

Let imagine that a set of branches with unknown parameters of a complex circuit can be allocated to a separate multipole network with inaccessible nodes (Fig. 5).

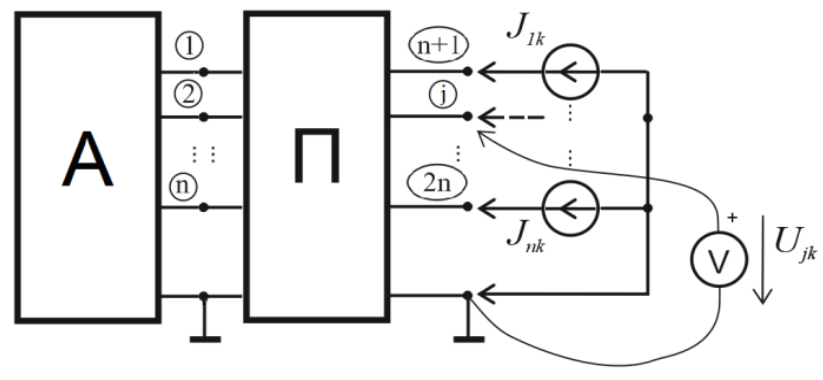

Figure 5. Circuit diagnosis with inaccessible nodes

Thus, there is an active diagnosed multipole network $\mathbf{A}$ with $\mathrm{n}$ independent nodes. Parameters of a subset of its elements which are incidental to nodes with numbers $(1, \ldots, n)$ are to be determined. To implement the diagnostic procedure it is possible to connect it to a complex circuit has, at least, $2 n$ independent nodes (numbered $1, \ldots, 2 n$ ).

And nodes with numbers $((n+1), \ldots, 2 n)$ are available to connect to the circuit test inputs, and to measure reactions (voltages). Measurements of node voltages $\left(U_{(n+1)}, \ldots U_{2 n}\right)$ can be considered as indirect measurement of node voltage $\left(U_{1}, \ldots, U_{n}\right)$. Compared with the principal model the test sources and the measurements are a certain distance away from the branches of the diagnosed multipole network (measured from the graph of circuit). It is clearly, that concerning to the nodes $((n+1), \ldots, 2 n)$ it is easy to define a matrix of input and transmission parameters, but elements the diagnosed parameters in the analytical expressions of the matrix will appear in the form of elements of rational functions.

If $\mathbf{Y}^{n}$ - the nodal admittance matrix of diagnosed multipole network with size $n \times n$, then $\mathbf{Y}_{S}=\left[\begin{array}{ll}\mathbf{Y}_{11} & \mathbf{Y}_{12} \\ \mathbf{Y}_{21} & \mathbf{Y}_{22}\end{array}\right]$ is the nodal admittance matrix of complex circuit with size $2 n \times 2 n$. Complete system of equations of interconnected diagnosed and passive circuits for $n$ experiments obtained in the form:

$$
\left[\begin{array}{cc}
\left(\mathbf{Y}^{n}+\mathbf{Y}_{11}\right) & \mathbf{Y}_{12} \\
\mathbf{Y}_{21} & \mathbf{Y}_{22}
\end{array}\right]\left[\begin{array}{l}
\mathbf{U}_{R}^{n} \\
\mathbf{U}_{R}^{m}
\end{array}\right]=\left[\begin{array}{l}
\mathbf{J}_{R}^{n} \\
\mathbf{J}_{R}^{m}
\end{array}\right],
$$

where are:

$\mathbf{U}_{\mathrm{R}}{ }^{n}$ - the square voltages matrix for the first $n$ nodes,

$\mathbf{U}_{\mathrm{R}}{ }^{s}$ - the square matrix or the voltages measured at the nodes with numbers $((n+1), \ldots, 2 n)$

$\mathbf{J}_{\mathrm{R}}{ }^{n}$ - the square matrix of internal (unknown) sources of the active multipole network (all column are indentical), $\mathbf{J}_{\mathbf{R}}{ }^{s}$ - the square matrix vector of the test sources.
Let from the 2nd row of the matrix (6) express $\mathbf{U}_{\mathrm{R}}{ }^{n}$ :

$$
\mathbf{U}_{\mathrm{R}}{ }^{n}=\mathbf{Y}_{21}{ }^{-1}\left(\mathbf{J}_{\mathrm{R}}{ }^{s}-\mathbf{Y}_{22} \mathbf{U}_{\mathrm{R}}{ }^{s}\right) \text {, }
$$

and substitute in the 1-st row of the matrix equation:

$$
\left(\mathbf{Y}^{n}+\mathbf{Y}_{11}\right) \mathbf{Y}_{21}{ }^{-1}\left(\mathbf{J}_{\mathrm{R}}{ }^{s}-\mathbf{Y}_{22} \mathbf{U}_{\mathrm{R}}{ }^{s}\right)=-\mathbf{Y}_{12} \mathbf{U}_{\mathrm{R}}{ }^{s}+\mathbf{J}_{\mathrm{R}}{ }^{n} .
$$

If at the open circuit $\mathbf{J}_{\mathrm{R}}{ }^{s}=\mathbf{0}, \quad \mathbf{U}_{\mathrm{R}}{ }^{s}=\mathbf{U}_{\mathrm{R} 0}$, we have the expression:

$$
\left(\mathbf{Y}^{n}+\mathbf{Y}_{11}\right) \mathbf{Y}_{21}{ }^{-1}\left(-\mathbf{Y}_{22} \mathbf{U}_{\mathrm{R} 0}\right)=-\mathbf{Y}_{12} \mathbf{U}_{\mathrm{R} 0}+\mathbf{J}_{\mathrm{R}}{ }^{n} .
$$

Now we can exclude $\mathbf{J}^{n}$ :

$$
\left(\mathbf{Y}^{n}+\mathbf{Y}_{11}\right) \mathbf{Y}_{21}{ }^{-1}\left(\mathbf{J}_{\mathrm{R}}{ }^{s}+\mathbf{Y}_{22}\left(\mathbf{U}_{\mathrm{R} 0^{-}} \mathbf{U}_{\mathrm{R}}^{s}\right)\right)=\mathbf{Y}_{12}\left(\mathbf{U}_{\mathrm{R} 0^{-}} \mathbf{U}_{\mathrm{R}}{ }^{s}\right) .
$$

Let introduce the notation:

$$
\begin{gathered}
\mathbf{U}_{\mathrm{R}}=\mathbf{Y}_{21}^{-1}\left(\mathbf{J}_{\mathrm{R}}{ }^{s}+\mathbf{Y}_{22}\left(\mathbf{U}_{\mathrm{R} 0^{-}} \mathbf{U}_{\mathrm{R}}{ }^{s}\right)\right), \\
\mathbf{J}_{\mathrm{R}}=\mathbf{Y}_{12}\left(\mathbf{U}_{\mathrm{R} 0^{-}} \mathbf{U}_{\mathrm{R}}{ }^{s}\right),
\end{gathered}
$$

And similarly (3) finally we get the expression:

$$
\mathbf{Y}^{n}=\mathbf{J}_{\mathrm{R}} \mathbf{U}_{\mathrm{R}}^{-1}-\mathbf{Y}_{11}
$$

\subsection{Example 3}

Let's consider the circuit showed at Fig.6. The diagnosed active multipole network $\mathbf{A}$ is represented by the set of branches $\mathbf{N}=\{4,3,7,12,13\}$ incidental to unaccessible nodes $1,2,3$; and measuring voltages are at the accessible nodes with numbers $4,5,6$.

It is meant that in a nominal mode values of all branch conductivities are: $y_{1} \ldots y_{13}=1$. The matrix of known parameters $\mathbf{Y}_{\mathbf{s}}$ appears in the form of:

$$
\mathbf{Y}_{S}=\left[\begin{array}{ll}
\mathbf{Y}_{11} & \mathbf{Y}_{12} \\
\mathbf{Y}_{21} & \mathbf{Y}_{22}
\end{array}\right]=\left[\begin{array}{l}
{\left[\begin{array}{rrr}
2 & -1 & 0 \\
-1 & 2 & 0 \\
0 & 0 & 1
\end{array}\right]} \\
{\left[\begin{array}{rrr}
0 & -1 & 0 \\
0 & 0 & -1 \\
-1 & 0 & 0
\end{array}\right]\left[\begin{array}{rrr}
0 & 0 & -1 \\
-1 & 0 & 0 \\
0 & -1 & 0
\end{array}\right]} \\
{\left[\begin{array}{ccc}
3 & -1 & -1 \\
-1 & 3 & -1 \\
-1 & -1 & 4
\end{array}\right]}
\end{array}\right]
$$

As well as [3], by a fault, here it is meant any changes in the values of elements of the $(3+1)$-pole circuit $A$ with respect to theirs nominal values.

Measured results of voltage for open circuit in a nominal mode have to be equal:

$$
\mathbf{U}_{0 \text { nom }}=\left[\begin{array}{c}
U_{40} \\
U_{50} \\
U_{60}
\end{array}\right]=\left[\begin{array}{c}
9.45 \\
4.38 \\
10.0
\end{array}\right] .
$$

Experimentally obtained data are:

$$
\mathbf{U}_{R 0}=\left[\begin{array}{l}
U_{40} \\
U_{50} \\
U_{60}
\end{array}\right]=\left[\begin{array}{l}
7.53 \\
3.37 \\
8.44
\end{array}\right],
$$

and for determination of 3-pole A parameters experiments 
are made. Test inputs $\mathbf{J}_{\mathrm{R}}{ }^{s}$ and test measuring voltages $\mathbf{U}_{\mathrm{R}}{ }^{s}$ and are:

$$
\mathbf{J}_{R}^{s}=\left[\begin{array}{ccc}
100 & 0 & 0 \\
0 & 100 & 0 \\
0 & 0 & 100
\end{array}\right], \mathbf{U}_{R}^{s}=\left[\begin{array}{ccc}
81.70 & 54.40 & 44.78 \\
50.23 & 75.22 & 39.57 \\
45.69 & 44.64 & 57.77
\end{array}\right]
$$

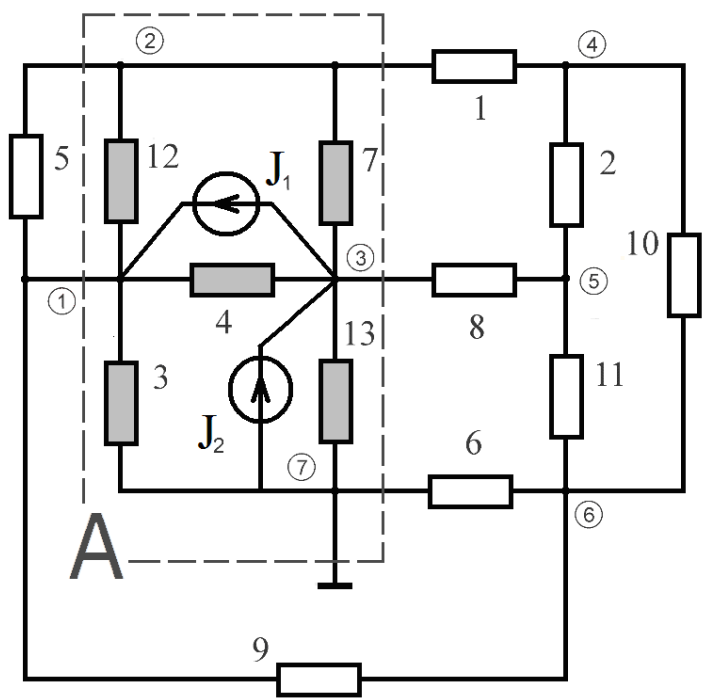

Figure 6. Circuit for Example 3

Let do further calculations:

$$
\begin{gathered}
\mathbf{U}_{\mathrm{R}}=\mathbf{Y}_{21}{ }^{-1}\left(\mathbf{J}_{\mathrm{R}}{ }^{s}+\mathbf{Y}_{22}\left(\mathbf{U}_{\mathrm{R} 0^{-}} \mathbf{U}_{\mathrm{R}}{ }^{s}\right)\right)= \\
=\left[\begin{array}{rrr}
27.92 & 26.08 & 23.87 \\
38.35 & 32.56 & 26.22 \\
29.31 & 32.48 & 22.02
\end{array}\right] ; \\
\mathbf{J}_{\mathrm{R}}=\mathbf{Y}_{12}\left(\mathbf{U}_{\mathrm{R} 0^{-}} \mathbf{U}_{\mathrm{R}}{ }^{s}\right)=\left[\begin{array}{lll}
37.25 & 36.20 & 49.33 \\
74.17 & 46.87 & 37.25 \\
46.91 & 71.85 & 36.20
\end{array}\right]
\end{gathered}
$$

The required conductance matrix is:

$$
\begin{gathered}
\mathbf{Y}^{n}=\mathbf{J}_{\mathrm{R}} \mathbf{U}_{\mathrm{R}}^{-1}-\mathbf{Y}_{11}= \\
=\left[\begin{array}{ccc}
3.4473 & -1.1527 & -1.1014 \\
-1.2254 & 2.6426 & -1.4241 \\
-1.0148 & -1.5028 & 3.5335
\end{array}\right] \approx\left[\begin{array}{ccc}
3.45 & -1.19 & -1.05 \\
-1.19 & 2.64 & -1.46 \\
-1.05 & -1.46 & 3.53
\end{array}\right]
\end{gathered}
$$

Thus as in a nominal mode all of $y_{\mathrm{k}}=1(k=4,3,7,12,13)$, their values received deviations:

$$
\begin{gathered}
\mathbf{Y}^{n}-\mathbf{Y}_{\text {nom }}^{n}=\left[\begin{array}{ccc}
\left(y_{3}+y_{4}+y_{12}\right) & -y_{12} & -y_{4} \\
-y_{12} & \left(y_{7}+y_{12}\right) & -y_{7} \\
-y_{4} & -y_{7} & \left(y_{4}+y_{7}+y_{13}\right)
\end{array}\right]- \\
-\left[\begin{array}{ccc}
3.45 & -1.19 & -1.05 \\
-1.19 & 2.64 & -1.46 \\
-1.05 & -1.46 & 3.53
\end{array}\right]=\left[\begin{array}{ccc}
0.45 & 0.19 & 0.05 \\
0.19 & 0.64 & 0.46 \\
0.05 & 0.46 & 0.53
\end{array}\right],
\end{gathered}
$$

$$
\begin{gathered}
\Delta y_{3}=0.21 ; \quad \Delta y_{4}=0.05 ; \quad \Delta y_{7}=0.46 ; \\
\Delta y_{12}=0.19 ; \quad \Delta y_{13}=0.02 .
\end{gathered}
$$

\section{Conclusion}

The formalization of tasks of circuit test diagnosing presented in the article has great opportunities for further development. Some directions of further researches are designated in [8].

\section{Acknowledgements}

This work was partly financially supported by the Russian Foundation Basic Researches, grant № 13-08-00924.

\section{REFERENCES}

[1] Bercowitz R.S. Conditions for network-element-value solvability. - IRE Trans. On Circuit Theory, 1962, vol.9, March, p. 24-29.

[2] Ahrikencheikh, Cherif, U.S. Patent 7,103,856 (2004) Determining points of maximum deflection of a printed circuit board under test.

[3] Wei Liao, Jingao Liu., Research on k-fault diagnosis and testability in analog circuit.- WSEAS Transactions On Electronics, 2008, vol. 5, Issue 10, pp.417-426.

[4] Zhang Wei; Xu Aiqiang; Chen Zhenlin, Fault dictionary method in analog circuits based on node voltage sensitivity weight sequence, Journal of Electronic Measurement and Instrument,Vol.20, no.4, ,pp.46-49.

[5] G. Zhen; J. Savir, Coefficient-based test of parametric faults in analog circuits, IEEE Trans. Instrum. Mea, Vol 55 , No.1, 2006, pp. $150-157$

[6] Demirchjan K.S., Butyrin P.A., Modeling and Computer Calculation of the Circuits. High School Publishers, Moskow (1988). (in Russian).

[7] Kinsht N.V., Gerasimova G.N., Katz M.A. Diagnostics of Electrical Circuits. Energoatomizdat Publishers, Moskow (1983) (in Russian).

[8] Kinsht N.V., Petrun'ko N.N., Electrical Circuits and System Diagnostics, Dalnauka Publishers, Vladivostok, (2013) (in Russian). 